\title{
Long-Term Structural Effects of Retinal Photocoagulation in Diabetic Retinopathy
}

\author{
Pedro Manuel Baptista ${ }^{1 *}$, Ana Ambrósio Marta ${ }^{1,2}$, João Heitor \\ Marques $^{1}$, André Ferreira ${ }^{1,3}$, Diana José ${ }^{1}$, Daniel Almeida ${ }^{1}$, António \\ Ribeiro $^{1,2}$ and Irene Barbosa ${ }^{1,2}$ \\ ${ }^{1}$ Ophthalmology Department, Centro Hospitalar Universitário do Porto, Portugal \\ ${ }^{2}$ Instituto de Clências Biomédicas Abel Salazar, Universidade do Porto, Potugal \\ ${ }^{3}$ Department of Biomedicine, Unit of Anatomy, Faculty of Medicine of the University \\ of Porto, porto, Portugal \\ *Corresponding Author: Pedro Manuel Baptista, Ophthalmology Department, \\ Centro Hospitalar Universitário do Porto, Porto, Portugal.
}

Received: February 09, 2021

Published: February 19, 2021

(C) All rights are reserved by Pedro Manuel

Baptista., et al.

\begin{abstract}
Background and Objective: Pan-retinal photocoagulation (PRP) have potential structural deleterious effects in retinal and choroidal tissues. To assess the long-term retinal and choroidal status after PRP and to correlate the results with treatment strategies.

Design/Materials and Methods: Observational cross-sectional study including eyes with diabetic retinopathy who underwent PRP, with or without macular treatments.

Results: 227 eyes with a mean time since PRP of $10.0 \pm 7.2$ years. In a multivariable model, the only treatment characteristics with predictive value were the macular laser treatment with modified grid for worse BCVA, the higher number of laser spots for higher CT and GCLV and the laser treatment with Argon for lower FNFLT.

Conclusion: This study demonstrates long-term stability in the BCVA and choroidal and retinal structural parameters with only mild correlation with treatment strategies, highlighting the role of PRP in the management of diabetic retinopathy in an era with evolving less aggressive laser options.
\end{abstract}

Keywords: Pan-Retinal Photocoagulation (PRP); Proliferative Diabetic Retinopathy (pDR); BCVA

\section{Background and Objective}

Pan-retinal photocoagulation (PRP) has become the Gold Standard for the treatment of proliferative diabetic retinopathy (pDR) since 1976, when the Diabetic Retinopathy Study Research Group [1] demonstrated a reduction in the rate of visual loss with laser treatment. Thereafter, several analyses [2-5] were conducted by the same group in order to evaluate the structural and functional results and, despite having demonstrated its effectiveness, they highlighted the harmful potential in the retinal and choroidal tissues with consequent functional repercussion. However, data regarding the real effects of PRP in retinal and choroidal tissues have been limited due to small populations, short follow-up times and evaluation of treatments with non-standardized parameterizations.

With the increase in the average life expectancy and the increase in the prevalence of RD in increasingly younger patients, now and in the future, more diabetic patients with PRP will be in systemic conditions compatible with an active life. Additionally, the evolution of conventional lasers to the various multispot systems, with possibilities of reducing exposure times and using different wavelengths with different absorption spectra, is increasing the number

Citation: Pedro Manuel Baptista., et al. "Long-Term Structural Effects of Retinal Photocoagulation in Diabetic Retinopathy". Acta Scientific Ophthalmology 4.3 (2021): 62-71. 
of treatment strategies, decreasing the potential for harm [6]. For these reasons, this theme will take on particular relevance.

Our group selected a group of these patients and subjected them to structural tests in order to quantify the potential deleterious effects and to relate the results with clinical factors and treatment strategies looking for predictors of prognosis.

\section{Patients/Materials and Methods Design}

Observational cross-sectional study set in the Ophthalmology Department, Centro Hospitalar e Universitário do Porto, Porto, Portugal, a tertiary center. The study adhered to the tenets of the Declaration of Helsinki.

\section{Population}

Eyes from diabetic patients diagnosed with DR and submitted to PRP with or without macular grid and/or focal photocoagulation in the posterior pole. Exclusion criteria were the presence of concurrent retinal vascular diseases, macular hole history, other concurrent macular diseases or retinal detachment, optic nerve diseases other than glaucoma, history of amblyopia and impaired ability to perform tests due to media opacities, ocular motricity problems or neurological/psychiatric status.

\section{Laser treatments}

The treatments were performed with Argon (514 nm) (Coherent $^{\circledR}$ ) and Double frequency nd:YAG monospot (nd:YAGII) (532 nm) $\left(\right.$ ZEISS $^{\circledR}$ ) and multispot (Valon ${ }^{\circledR}$ ) lasers. The timing most frequently used was $100 \mathrm{~ms}$ with the Argon laser and 20 - $30 \mathrm{~ms}$ with the nd:YAGII. The spot size used was 50 or $100 \mu \mathrm{m}$ in the macular grid or focal photocoagulation at the posterior pole, 200 to $250 \mu \mathrm{m}$ in the photocoagulation adjacent to the vascular arcades and 200 to 500 $\mu \mathrm{m}$ in the periphery, depending, among other factors, on the media opacity and the magnification of the contact lenses used. The power was selected in order to allow visualization of the whitish marks of the impacts on the retina.

\section{Data gathering}

Demographic and clinical data were obtained through analysis of patients' clinical records. Data was collected regarding: age; gender; type and duration of diabetes $(\mathrm{tD})$; duration of the diabetic retinopathy (tDR); lens status; history of diabetic macular edema (DME) and/or intravitreal treatments of anti-vascular endothelial growth factor (VEGF) or corticosteroids; concomitance of epiretinal membrane with significant horizontal traction (ERM); history of glaucoma or ocular hypertension (OHT); previous vitreo-retinal (VR) surgery; history of peripheral retinal cryoablation (PRC). Best corrected visual acuity was measured at a distance of 6 meters, on the Snellen scale (BCVA) and then presented in decimal scale for an intuitive analysis. The presence of systemic hypertension (HTA) and insulin treatment were also recorded.

Retinal photocoagulation characteristics were obtained by analysing all clinical records, retinographs, and previous angiographies, also contained in clinical processes, always by the same specialist. Regarding PRP, three patterns were considered, according to the predominance of the following aspects: non-confluent, when the marks of the various impacts were evidenced individually, with spacing between them greater than one mark size; confluent, when this spacing was equal or less than one mark size; very confluent, when there was coalescence of the various marks. Additionally, the quantification of the total treated area was calculated based on the sum of the various impacts made in the different photocoagulation sessions, with the reduction of the various spot sizes to a $250 \mu \mathrm{m}$ spot size area. Photocoagulation within the posterior pole was performed divided in two categories (modified ETDRS-style): Focal and in the form of a macular temporal horseshoe or modified macular grid (Modified grid), always preserving the papillomacular bundle. Type of laser used and time since PRP (tPRP) were also analysed.

The evaluation of the retinal and choroidal structure was carried-out using spectral domain optical coherence tomography (SD-OCT, Heidelberg Spectralis ${ }^{\circledR}$ ), analysing the mean values of the subfoveal choroidal thickness (CT, $\mu \mathrm{m})$, macular ganglion cell layer volume (GCLV, $\mathrm{mm}^{3}$ ), $6 \mathrm{~mm}$ ETDRS ring), foveal nerve fiber layer thickness (FNFLT, $\mu \mathrm{m}$, central $1 \mathrm{~mm}$ of the ETDRS ring), optic disc nerve fiber layer thickness (ODNFLT, $\mu \mathrm{m}$, peripapillary 3.5 $\mathrm{mm}$ ring) and the presence of foveal intraretinal cists at the time of evaluation.

\section{Statistical analysis}

All analysis were performed using the SPSS ${ }^{\circledR}$ and Stata ${ }^{\circledR}$ software's. Normality of the data was tested with the Shapiro-Wilk and Kolmogorov-Smirnov tests. Levene's test was used to look for homogeneity of variances. When parametric analysis could be ap- 
plied, the Student t-test was used to compare the variables. When nonparametric tests were needed, the Wilcoxon rank-sum test was applied. The $\chi^{2}$ was used to compare nominal and ordinal variables. Spearman's rank correlation coefficient was used to correlate variables.

Candidate predictors in multivariable analysis were the treatment characteristics and patient's demographic and clinical factors. Predictors were screened to identify associations with BCVA, CT, GCLV, FNFLT and ODNFLT at the $\mathrm{p}<0.25$ level. Predictors meeting this criterion were included in stepwise backward analysis in which $\mathrm{p}<0.05$ served as the criterion for retention into the full model - exceptions were made when clinically appropriate.

All values are shown as mean \pm standard deviation unless otherwise specified. All p-values (p) were 2-sided, and p-values $<0.05$ were considered significant.

\section{Results}

Demographics and general descriptions

This study included 227 eyes of 123 diabetic patients who underwent PRP. Table 1 summarizes clinical and demographic characteristics. Mean age was $64.5 \pm 11.3$ years, 77 (63\%) patients were male, mean tD and tDR were $29.3 \pm 10.7$ years and $18.1 \pm 9.1$ years, respectively, and mean tPRP was $10.0 \pm 7.2$ years. History of DME and intravitreal treatments were present in 59 (26\%) and 29 (13\%) eyes, respectively. ERM with significant horizontal traction was present in 52 (23\%) eyes, OHT or glaucoma were present in 63 (27\%) eyes, and history of PRC in 29 (13\%) eyes. Ninety-five (77\%) patients were under insulin therapy and 54 (44\%) presented HTA.

\begin{tabular}{|c|c|c|c|c|}
\hline & All sample & T1 & T2 & \multirow{3}{*}{$\begin{array}{c}\text { T1 VS } \\
\text { T2 } \\
\text { (p) }\end{array}$} \\
\hline & 227 eyes & 67 eyes & 160 eyes & \\
\hline & $\begin{array}{c}123 \\
\text { patients }\end{array}$ & $\begin{array}{c}35 \\
\text { patients }\end{array}$ & $\begin{array}{c}\mathbf{8 8} \\
\text { patients }\end{array}$ & \\
\hline Age (years) & $64.5 \pm 11.3$ & $\begin{array}{c}54.1 \pm \\
10.5 \\
\end{array}$ & $\begin{array}{c}68.6 \pm \\
8.7\end{array}$ & $\begin{array}{c}< \\
0.001 \\
\end{array}$ \\
\hline Gender (male) & 77 (63\%) & $\begin{array}{c}19 \\
(54 \%)\end{array}$ & $\begin{array}{c}58 \\
(66 \%) \\
\end{array}$ & 0.23 \\
\hline tD (years) & $29.3 \pm 10.7$ & $\begin{array}{c}34.8 \pm \\
9.5\end{array}$ & $\begin{array}{c}27.1 \pm \\
10.3\end{array}$ & $\begin{array}{c}< \\
0.001 \\
\end{array}$ \\
\hline tDR (years) & $18.1 \pm 9.1$ & $\begin{array}{c}22.6 \pm \\
9.3\end{array}$ & $\begin{array}{c}15.9 \pm \\
8.2\end{array}$ & $\begin{array}{c}< \\
0.001\end{array}$ \\
\hline tPRP (years) & $10.0 \pm 7.2$ & $\begin{array}{c}13.1 \pm \\
8.2\end{array}$ & $8.7 \pm 6.2$ & $\begin{array}{c}< \\
0.001\end{array}$ \\
\hline
\end{tabular}

\begin{tabular}{|c|c|c|c|c|}
\hline $\begin{array}{l}250 \mu \mathrm{m} \text { Spots } \\
(\mathrm{nr})\end{array}$ & $5543 \pm 2134$ & $\begin{array}{c}6304 \pm \\
2593\end{array}$ & $\begin{array}{c}5182 \pm \\
1779\end{array}$ & $\begin{array}{c}< \\
0.001\end{array}$ \\
\hline \multicolumn{5}{|c|}{ Lens status, n (\% within group) } \\
\hline $\begin{array}{l}\text { Transparent/ } \\
\text { pseudophakic }\end{array}$ & $131(57.7 \%)$ & $\begin{array}{c}37 \\
(55.2 \%)\end{array}$ & $\begin{array}{c}94 \\
(58.8 \%)\end{array}$ & \multirow{2}{*}{0.62} \\
\hline Cataract & $96(42.3 \%)$ & $\begin{array}{c}30 \\
(44.8 \%)\end{array}$ & $\begin{array}{c}66 \\
(41.2 \%) \\
\end{array}$ & \\
\hline \multicolumn{5}{|c|}{ Ophthalmological history, n (\% within group) } \\
\hline DME history & $59(26 \%)$ & $\begin{array}{c}14 \\
(21 \%) \\
\end{array}$ & $\begin{array}{c}45 \\
(29 \%) \\
\end{array}$ & 0.26 \\
\hline $\begin{array}{l}\text { Foveal } \\
\text { intraretinal } \\
\text { cists }\end{array}$ & $52(23 \%)$ & $8(12 \%)$ & $\begin{array}{c}44 \\
(28 \%)\end{array}$ & 0.011 \\
\hline $\begin{array}{l}\text { Intravitreal } \\
\text { treatments }\end{array}$ & $29(13 \%)$ & $4(6 \%)$ & $\begin{array}{c}25 \\
(16 \%) \\
\end{array}$ & 0.051 \\
\hline $\begin{array}{l}\text { ERM with } \\
\text { traction }\end{array}$ & $52(23 \%)$ & $\begin{array}{c}11 \\
(17 \%)\end{array}$ & $\begin{array}{c}43 \\
(27 \%) \\
\end{array}$ & 0.09 \\
\hline $\begin{array}{l}\text { OHT/ } \\
\text { Glaucoma }\end{array}$ & $63(27 \%)$ & $\begin{array}{c}10 \\
(15 \%)\end{array}$ & $\begin{array}{c}53 \\
(33 \%)\end{array}$ & 0.006 \\
\hline VR surgery & $32(14 \%)$ & $\begin{array}{c}14 \\
(21 \%) \\
\end{array}$ & $\begin{array}{c}18 \\
(11 \%) \\
\end{array}$ & 0.06 \\
\hline PRC & $29(13 \%)$ & $\begin{array}{c}12 \\
(18 \%) \\
\end{array}$ & $\begin{array}{c}17 \\
(11 \%) \\
\end{array}$ & 0.16 \\
\hline \multicolumn{5}{|c|}{ Systemic history, n (\% within group) } \\
\hline Insulin & $95(77 \%)$ & $\begin{array}{c}31 \\
(89 \%) \\
\end{array}$ & $\begin{array}{c}64 \\
(73 \%) \\
\end{array}$ & 0.059 \\
\hline HTN & $54(44 \%)$ & $\begin{array}{c}10 \\
(29 \%)\end{array}$ & $\begin{array}{c}44 \\
(50 \%)\end{array}$ & 0.031 \\
\hline
\end{tabular}

Table 1: Clinical and demographic data: overall and per type of diabetes analysis.

Footnotes: T1: Type 1 diabetic patients, T2: Type 2 diabetic patients, $\mathrm{tD}$ : Time since diabetes diagnosis, $\mathrm{tD}$ : Time since diabetic retinopathy diagnosis, tPRP: Time since panretinal photocoagulation, DME: Diabetic macular edema; ERM: Epiretinal membrane; OHT: Ocular hypertension, VR: Vitreo-retinal, PRC: Peripheral retinal cryoablation, HTN: Systemic hypertension.

\section{Treatment characteristics}

The most common type of laser used was the Argon $(62 \%, n=$ $142)$, the most common pattern was the confluent $(70 \%, n=156)$ and the majority of the eyes $(87 \%, n=195)$ underwent posterior pole laser treatments. Table 2 summarizes PRP treatment data. 


\begin{tabular}{|c|c|c|c|c|}
\hline & All sample & T1 & T2 & \multirow{3}{*}{$\begin{array}{l}\text { T1 VS } \\
\text { T2 (p) }\end{array}$} \\
\hline & 227 eyes & 67 eyes & 160 eyes & \\
\hline & 123 patients & $\begin{array}{c}35 \\
\text { patients }\end{array}$ & $\begin{array}{c}88 \\
\text { patients }\end{array}$ & \\
\hline \multicolumn{5}{|c|}{ Type of laser, $n$ (\% within group) } \\
\hline Argon & $142(62 \%)$ & $49(73 \%)$ & $93(58 \%)$ & \multirow{2}{*}{0.033} \\
\hline nd:YAGII & $85(38 \%)$ & $18(27 \%)$ & $67(42 \%)$ & \\
\hline \multicolumn{5}{|c|}{ Pattern of PRP, n (\% within group) } \\
\hline $\begin{array}{l}\text { Non } \\
\text {-confluent }\end{array}$ & $18(8 \%)$ & $3(4 \%)$ & $15(10 \%)$ & \multirow{3}{*}{0.27} \\
\hline Confluent & $156(70 \%)$ & $46(69 \%)$ & $110(70 \%)$ & \\
\hline $\begin{array}{l}\text { Very } \\
\text { confluent }\end{array}$ & $49(22 \%)$ & $18(27 \%)$ & $31(20 \%)$ & \\
\hline \multicolumn{5}{|c|}{ Macular treatments, $\mathbf{n}$ (\% within group) } \\
\hline $\begin{array}{l}\text { No } \\
\text { treatment }\end{array}$ & $29(13 \%)$ & $14(21 \%)$ & $15(10 \%)$ & \multirow{3}{*}{0.07} \\
\hline Focal & $89(40 \%)$ & $24(36 \%)$ & $65(41 \%)$ & \\
\hline $\begin{array}{l}\text { Modified } \\
\text { grid }\end{array}$ & $106(47 \%)$ & $29(43 \%)$ & 77 (49\%) & \\
\hline
\end{tabular}

Table 2: Photocoagulation treatment data: overall and per type of diabetes analysis.

Footnotes: T1: Type 1 diabetic patients, T2: Type 2 diabetic patients, Argon: Argon laser (514 nm, Coherent $^{\circledR}$ ), nd:YAGII: Double frequency nd:YAG (532 nm) monospot (ZEISS $\left.{ }^{\circledR}\right)$ and multispot $\left(\right.$ Valon $\left.^{\circledR}\right)$ lasers.

\section{BCVA}

Mean BCVA was $0.57 \pm 0.29$. Factors associated with worse BCVA included type 2 diabetes, presence of cataract, foveal cysts and tractional ERM and history of intravitreal treatments and macular laser therapy, particularly modified grid.

\section{Retinal and choroidal structural analysis}

Table 3 and 4 summarize retinal and choroidal structural parameters by type and pattern of treatments and by clinical factors, respectively.

\section{Comparison by type of diabetes}

The higher prevalence of Argon laser in type 1 diabetic patients ( $p=0.033$ ) was the only significant difference found in treatment characteristics between groups. All comparisons are described in table 1 and 2.

\section{Multivariable regression analysis}

A multivariable regression model was built to determine potential independent predictors of the visual and structural outcomes and adjust for potential confounders (Table 5). Older age, shorter tD, macular laser treatment with modified grid (vs focal pattern) and presence of cataract, foveal intraretinal cysts and tractional ERM were independent predictors of worse BCVA. Higher number of laser spots, presence of foveal intraretinal cysts and the lack of tractional ERM were independent predictors of higher CT. Higher number of laser spots, history of VR surgery and younger age were independent predictors for higher GCLV. Laser treatment with nd:YAGII (vs Argon), presence of foveal intraretinal cysts and history of intravitreal treatments were independent predictors of higher FNFLT. The presence of cataract was the only independent predictor of higher ODNFLT.

\section{Discussion and Conclusion}

Overall, average BCVA in the present study was compatible with daily life activities. Predictably, cataract and macular alterations were associated to worse results. After the adjustment for the possible confounders, the only associated treatment predictor of worse BCVA was macular modified grid pattern as macular treatment. Back in 2007 [7], the modified ETDRS direct/grid photocoagulation technique was reported to be structurally more effective at 12 months in the treatment of non-center involved clinically significant DME when compared with a strategy in which small mild burns were placed throughout the macula, whether or not edema was present. Additionally, the more aggressive strategy did not affect significantly visual acuity [7]. Another report proved [8] the 12-month stability in visual acuity from baseline after modified ETDRS style (focal/grid) photocoagulation in eyes with noncenter involved clinically significant DME. Although It is expected that eyes with previous modified grid treatment were those with more aggressive DME, which is itself a factor directly associated to long-term decrease of BCVA, the BCVA absolute values on both patterns of macular laser treatment are, in average, good and compatible with daily life activities in the present study. When comparing the modified ETDRS direct/grid photocoagulation technique with novel technology associated techniques like the subthreshold micropulse diode laser [9] in center-involving, clinically significant DME, there was no significant difference in terms of visual acuities, despite better retinal sensitivity results with the novel approach. 
66

\begin{tabular}{|c|c|c|c|c|c|c|c|c|c|c|}
\hline & \multicolumn{2}{|c|}{ BCVA (decimal) } & \multicolumn{2}{|c|}{$\mathrm{CT}(\mu \mathrm{m})$} & \multicolumn{2}{|c|}{$\operatorname{GCLV}\left(\mathrm{mm}^{3}\right)$} & \multicolumn{2}{|c|}{ fRNFLT $(\mu \mathrm{m})$} & \multicolumn{2}{|c|}{ ppRNFLT $(\mu \mathrm{m})$} \\
\hline & Mean \pm SE & $\mathbf{p}$ & Mean \pm SE & $\mathbf{p}$ & Mean \pm SE & $\mathbf{p}$ & $\begin{array}{l}\text { Mean } \pm \\
\text { SE }\end{array}$ & $\mathbf{p}$ & Mean \pm SE & $\mathbf{p}$ \\
\hline \multicolumn{11}{|l|}{ Type of Laser } \\
\hline nd: YAGII (532 nm) & $0.58 \pm 0.30$ & 0.77 & $237.9 \pm 76.7$ & 0.07 & $1.1 \pm 0.2$ & 0.51 & $\begin{array}{c}19.4 \pm \\
13.4\end{array}$ & $<0.001$ & $96.8 \pm 14.3$ & 0.11 \\
\hline \multicolumn{11}{|l|}{ PRP pattern } \\
\hline $\begin{array}{l}\text { Laser Confluent, } \mathrm{n} \\
=156\end{array}$ & $0.59 \pm 0.29$ & 0.79 & $230.0 \pm 67.2$ & 0.69 & $1.5 \pm 0.3$ & 0.52 & $\begin{array}{c}16.3 \pm \\
10.6\end{array}$ & 0.38 & $96.3 \pm 21.8$ & 0.001 \\
\hline \multicolumn{11}{|l|}{$\begin{array}{l}\text { Macular } \\
\text { treatments }\end{array}$} \\
\hline Yes & $0.55 \pm 0.29$ & \multirow[t]{2}{*}{0.019} & $230.1 \pm 64.9$ & \multirow[t]{2}{*}{0.14} & $1.4 \pm 0.2$ & \multirow[t]{2}{*}{0.67} & $\begin{array}{c}16.6 \pm \\
11.0\end{array}$ & \multirow[t]{2}{*}{0.22} & $94.2 \pm 21.2$ & \multirow[t]{2}{*}{0.57} \\
\hline No & $0.69 \pm 0.29$ & & $210.7 \pm 74.6$ & & $1.0 \pm 0.2$ & & $14.0 \pm 5.4$ & & $91.8 \pm 16.9$ & \\
\hline \multicolumn{11}{|l|}{$\begin{array}{l}\text { Macular } \\
\text { treatment } \\
\text { pattern }\end{array}$} \\
\hline Focal & $0.60 \pm 0.26$ & \multirow[b]{2}{*}{0.024} & $222.9 \pm 61.7$ & \multirow[b]{2}{*}{0.16} & $1.02 \pm 0.2$ & \multirow[b]{2}{*}{0.36} & $16.2 \pm 8.9$ & \multirow[b]{2}{*}{0.66} & $92.2 \pm 15.0$ & \multirow[b]{2}{*}{0.23} \\
\hline Modified Grid & $0.51 \pm 0.31$ & & $236.2 \pm 67.1$ & & $1.7 \pm 0.4$ & & $\begin{array}{c}16.9 \pm \\
12.6\end{array}$ & & $95.9 \pm 25.2$ & \\
\hline
\end{tabular}

Table 3. Overall and per laser treatment subgroup analysis.

Footnotes: CT: Subfoveal choroidal thickness; CFT: Central foveal thickness; GCLV: Macular ganglion cell layer volume; fRNFLT: Foveal retinal nerve fiber layer thickness; ppRNFLT: Peripapillary retinal nerve fiber layer thickness; Argon: Argon laser (514 nm, Coherent ${ }^{\circ}$ ), nd:YAGII: Double frequency nd:YAG (532 nm) monospot (ZEISS $\left.{ }^{\circ}\right)$ and multispot (Valon ${ }^{\circ}$ ) lasers, PRP: Panretinal photocoagulation.

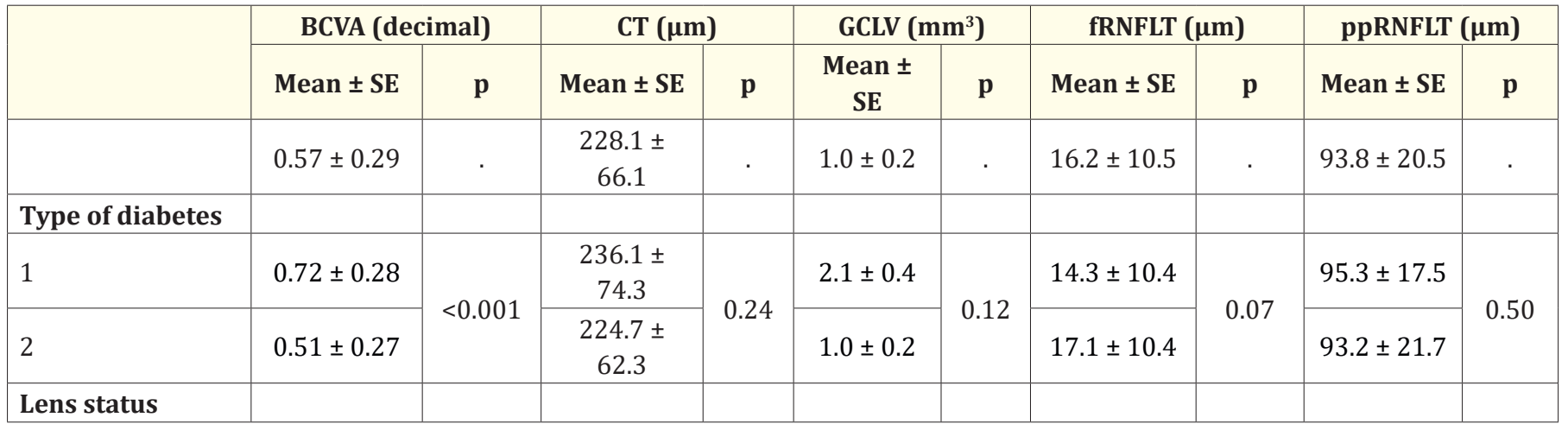

Citation: Pedro Manuel Baptista., et al. "Long-Term Structural Effects of Retinal Photocoagulation in Diabetic Retinopathy". Acta Scientific Ophthalmology 4.3 (2021): 62-71. 


\begin{tabular}{|c|c|c|c|c|c|c|c|c|c|c|}
\hline $\begin{array}{l}\text { Transparency + } \\
\text { Pseudophaquic }\end{array}$ & $0.63 \pm 0.29$ & \multirow{2}{*}{0.001} & $\begin{array}{c}223.8 \pm \\
61.9\end{array}$ & \multirow{2}{*}{0.26} & $1.6 \pm 0.3$ & \multirow{2}{*}{0.40} & $16.0 \pm 9.8$ & \multirow{2}{*}{0.74} & $90.5 \pm 15.9$ & \multirow{2}{*}{0.004} \\
\hline Cataract & $0.50 \pm 0.28$ & & $\begin{array}{c}233.9 \pm \\
71.5\end{array}$ & & $1.0 \pm 0.2$ & & $16.5 \pm 11.4$ & & $98.5 \pm 24.9$ & \\
\hline \multicolumn{11}{|l|}{ DME history } \\
\hline Yes & $0.54 \pm 0.29$ & \multirow{2}{*}{0.32} & $\begin{array}{c}241.0 \pm \\
74.3\end{array}$ & \multirow{2}{*}{0.08} & $1.0 \pm 0.2$ & \multirow{2}{*}{0.57} & $20.6 \pm 14.2$ & \multirow{2}{*}{$<0.001$} & $93.4 \pm 16.9$ & \multirow{2}{*}{0.85} \\
\hline No & $0.58 \pm 0.29$ & & $\begin{array}{c}223.5 \pm \\
62.6\end{array}$ & & $1.5 \pm 0.6$ & & $14.5 \pm 7.8$ & & $94.0 \pm 21.7$ & \\
\hline \multicolumn{11}{|l|}{$\begin{array}{l}\text { Foveal } \\
\text { intraretinal cists }\end{array}$} \\
\hline Yes & $0.50 \pm 0.28$ & \multirow{2}{*}{0.001} & $\begin{array}{c}241.6 \pm \\
85.4\end{array}$ & \multirow{2}{*}{0.09} & $1.1 \pm 0.2$ & \multirow{2}{*}{0.65} & $23.0 \pm 16.9$ & \multirow{2}{*}{$<0.001$} & $93.9 \pm 14.8$ & \multirow{2}{*}{0.98} \\
\hline No & $0.63 \pm 0.29$ & & $\begin{array}{c}224.0 \pm \\
58.8\end{array}$ & & $1.4 \pm 0.6$ & & $14.0 \pm 5.6$ & & $93.8 \pm 22.0$ & \\
\hline \multicolumn{11}{|l|}{$\begin{array}{l}\text { Intravitreal } \\
\text { treatments }\end{array}$} \\
\hline Yes & $0.40 \pm 0.28$ & \multirow{2}{*}{$<0.001$} & $\begin{array}{c}249.2 \pm \\
92.8\end{array}$ & \multirow{2}{*}{0.07} & $1.0 \pm 0.2$ & \multirow{2}{*}{0.69} & $24.7 \pm 18.2$ & \multirow{2}{*}{$<0.001$} & $93.1 \pm 12.6$ & \multirow{2}{*}{0.84} \\
\hline No & $0.60 \pm 0.29$ & & $\begin{array}{c}224.9 \pm \\
60.9\end{array}$ & & $1.4 \pm 0.5$ & & $14.8 \pm 7.7$ & & $93.9 \pm 21.5$ & \\
\hline \multicolumn{11}{|l|}{$\begin{array}{l}\text { ERM with } \\
\text { traction }\end{array}$} \\
\hline Yes & $0.44 \pm 0.29$ & \multirow{2}{*}{$<0.001$} & $\begin{array}{c}210.8 \pm \\
54.3\end{array}$ & \multirow{2}{*}{0.027} & $1.1 \pm 0.2$ & \multirow{2}{*}{0.64} & $17.5 \pm 8.1$ & \multirow{2}{*}{0.28} & $94.8 \pm 28.2$ & \multirow{2}{*}{0.69} \\
\hline No & $0.62 \pm 0.28$ & & $\begin{array}{c}233.6 \pm \\
68.7\end{array}$ & & $1.4 \pm 0.4$ & & $15.7 \pm 10.9$ & & $93.5 \pm 17.5$ & \\
\hline \multicolumn{11}{|l|}{ OHT/Glaucoma } \\
\hline Yes & $0.51 \pm 0.25$ & \multirow{2}{*}{0.032} & $\begin{array}{c}225.5 \pm \\
64.5\end{array}$ & \multirow{2}{*}{0.71} & $0.9 \pm 0.2$ & & $16.0 \pm 9.4$ & & $89.7 \pm 26.0$ & \\
\hline No & $0.60 \pm 0.30$ & & $\begin{array}{c}229.1 \pm \\
65.4\end{array}$ & & $1.5 \pm 0.4$ & 0.46 & $16.1 \pm 10.6$ & 0.94 & $95.4 \pm 17.8$ & 0.06 \\
\hline VR surgery & & & & & & & & & & \\
\hline Yes & $0.54 \pm 0.29$ & C & $\begin{array}{c}218.4 \pm \\
57.7\end{array}$ & n & $3.3 \pm 0.4$ & 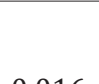 & $15.9 \pm 6.3$ & (0) & $87.6 \pm 15.8$ & 0 \\
\hline No & $0.58 \pm 0.29$ & 0.45 & $\begin{array}{c}229.4 \pm \\
67.5 \\
\end{array}$ & 0.39 & $1.0 \pm 0.2$ & 0.016 & $16.3 \pm 11.1$ & 0.83 & $94.9 \pm 21.1$ & 0.068 \\
\hline PRC & & & & & & & & & & \\
\hline Yes & $0.57 \pm 0.25$ & 0 & $\begin{array}{c}201.6 \pm \\
50.0\end{array}$ & & $3.5 \pm 0.4$ & & $12.2 \pm 4.0$ & & $94.9 \pm 38.1$ & 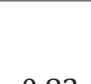 \\
\hline No & $0.58 \pm 0.30$ & 0.89 & $\begin{array}{c}232.3 \pm \\
68.5\end{array}$ & $0.0<3$ & $1.0 \pm 0.2$ & 0.012 & $16.9 \pm 11.1$ & 0.028 & $94.0 \pm 16.6$ & 0.82 \\
\hline
\end{tabular}

Table 4: Overall and per clinical and demographic subgroup analysis.

Footnotes: CT: Subfoveal choroidal thickness; CFT: Central foveal thickness; GCLV: Macular ganglion cell layer volume; fRNFLT: Foveal retinal nerve fiber layer thickness; ppRNFLT: Peripapillary retinal nerve fiber layer thickness; DME: Diabetic macular edema; ERM: Epiretinal membrane; OHT: ocular hypertension, VR: Vitreo-retinal, PRC: Peripheral retinal cryoablation. 


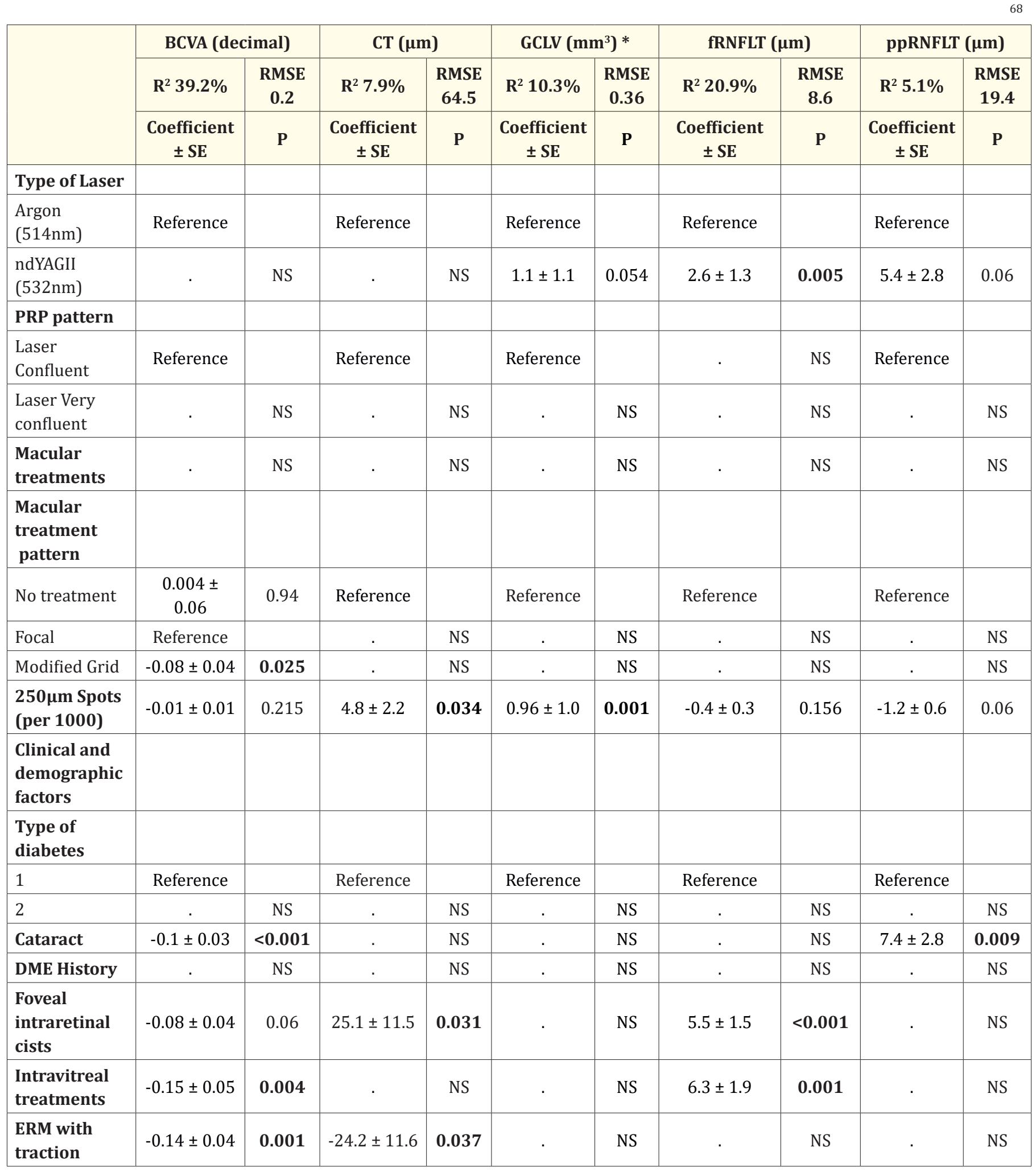




\begin{tabular}{|c|c|c|c|c|c|c|c|c|c|}
\hline $\begin{array}{l}\text { OHT/ } \\
\text { Glaucoma }\end{array}$ & . & NS & . & NS & . & NS & . & NS & NS \\
\hline VR surgery & . & NS & . & NS & $1.2 \pm 1.1$ & 0.02 & . & NS & NS \\
\hline PRC & . & NS & . & NS & . & NS & $-3.4 \pm 2.1$ & 0.11 & NS \\
\hline Age (years) & $\begin{array}{c}-0.01 \pm \\
0.001\end{array}$ & $<0.001$ & $-0.6 \pm 0.4$ & 0.125 & $0.99 \pm 1.0$ & 0.001 & . & NS & NS \\
\hline tD (years) & $\begin{array}{c}0.004 \pm \\
0.002\end{array}$ & 0.02 & $-0.7 \pm 0.5$ & 0.110 & . & NS & . & NS & NS \\
\hline tRD (years) & . & NS & & & . & NS & . & NS & NS \\
\hline tPRP (years) & . & NS & . & NS & . & NS & $0.2 \pm 0.1$ & 0.066 & NS \\
\hline
\end{tabular}

Table 5: Multivariate regression model.

*: A logarithmic transformation was performed for this variable. The coefficients are presented as $\exp (\mathrm{b})$.

Footnotes: CT: Subfoveal choroidal thickness; CFT: Central foveal thickness; GCLV: Macular ganglion cell layer volume; fRNFLT: Foveal retinal nerve fiber layer thickness; ppRNFLT: Peripapillary retinal nerve fiber layer thickness; Argon: Argon laser(514nm, Coherent ${ }^{\circ}$ ), nd:YAGII: Double frequency nd:YAG (532nm) monospot (ZEISS ${ }^{\circ}$ ) and multispot (Valon`) lasers, PRP: Panretinal photocoagulation; DME:

Diabetic macular edema; ERM: Epirretinal membrane; OHT: Ocular hypertension, VR: Vitreo-retinal, PRC: Peripheral retinal cryoablation; RMSE: Root Mean-Square Error, $\mathrm{tD}$ : Time since diabetes diagnosis, tD: Time since diabetic retinopathy diagnosis, tPRP: Time since panretinal photocoagulation.

The data in the present study proves the long-term stability of the BCVA after both PRP and ETDRS-style macular laser treatments (in center and non-center involved clinically significant DME) and encourages the use of the even less aggressive novel laser technologies existing today.

Within the structural analysis, the normality of the average values found in the 4 evaluated parameters should be emphasized, in patients with various co-morbidities and an average time of a decade since the beginning of treatment.

Regarding the subfoveal CT, it was reported [10] the association between higher values in the more advanced DR stages. Additionally, the idea of a decrease in the choroidal thickness in the peripheral treated areas associated to an increase in the macular region, due to remodeling in the choroidal homeostasis in the short term after PRP, was proven afterwards [11]. Despite lesser values reported with enhanced depth imaging mode (OCT) in younger healthy subjects [12], the absolute average results in the present study are similar to those reported recently [13]. Additionally, being the higher number of spots the only treatment related predictor of higher subfoveal CT and with only mild increase associated, this highlights the long-term stability of the subfoveal CT after PRP.

This study presented average values of both FNFLT and GCLV slightly higher than those reported in a recent proposed normative database [14]. The macular region retinal nerve fiber layer was reported to increase in the short-term [15] due to axonal edema but decrease thereafter since standard PRP with Argon laser [16]. The increased vascular permeability in the superficial vascular plexus associated to heat damage was reported to increase the thickness of the macular ganglion cell complex in the initial months, with subsequent return to baseline values at 12 months in one study [17] but not in another one [18]. According to the authors' knowledge, this is the first study reporting measurements on these retinal layers on a long-term basis. Although the idea of possible longterm significant decrease of inner retinal layers due to absorption of the edema and ganglion cell damage, the present study proves stability in both FNFLT and GCLV in a large sample after a decade, on average, since PRP. After the adjustment for the confounders, the argon laser was the only treatment factor predicting decreased FNFLT and a higher number of spots was the only treatment factor predicting higher GCLV. 
A study including 206 eyes [19] reported time-related decreased values in the ODNFLT measurements after an average of 2.5 years since PRP. The absolute average value measured in our sample is higher than the one described for both the normotension glaucoma group and the subgroup of patients with more than 3 years of follow-up since PRP in the reported study [19] and higher than the one recently described in a prospective study [20] 3 years after PRP. The ODNFLT results present in our sample are in line with a meta-analysis [21] which concluded a variable and non-significant effect of PRP. Additionally, there were no treatment factors predicting worse results in the present study.

Despite the longer tD, tDR and tPRP at a younger age and with longer life expectancy, and after the adjustment for the other clinical factors and treatment characteristics described above, type 1 diabetes didn't predict worse results in any of the evaluated outcomes. This reiterates a good risk-benefit ratio in carrying out these treatments at an early age in DR, known to be more aggressive in these patients.

The lack of baseline data or a control group could be understood as two limitations of the study. Regarding the first one, it would be impossible, since the current retinal imaging methods are not comparable to those existing when these patients were submitted to PRP. Regarding the second, as PRP is the gold standard since 1976, with no other approved therapeutic option, and non-treatment not being an option, it would always be impossible to make a comparison with a control group. The main strengths are the large sample size, the long time since PRP and the proportion of type 1 diabetic patients.

In summary, the present study demonstrates long-term stability in the BCVA and choroidal and retinal structural parameters evaluated, with only mild correlation with treatment strategies, despite the known retinal tissue aggression inherent to the procedure, Additionally, evaluating the structural effects of treatments performed with conventional lasers and with the known more aggressive strategies used in the past, the present study values the role of PRP as a safe strategy to treat this public health problem, in the present and future era of the modern laser options and new tailored treatment strategies, less aggressive to retinal tissue [22].

\section{Disclosure}

There is no financial, activity relationships or affiliations disclosures regarding any of the authors.

\section{Acknowledgments}

The authors want to acknowledge all the support granted by the head of the Ophthalmology department of Centro Hospitalar e Universitário do Porto, Prof. Dr. Pedro Menéres.

\section{Bibliography}

1. Preliminary report on effects of photocoagulation therapy. The Diabetic Retinopathy Study Research Group". American Journal of Ophthalmology 81.4 (1976): 383-396.

2. Early photocoagulation for diabetic retinopathy. "ETDRS report number 9. Early Treatment Diabetic Retinopathy Study Research Group". Ophthalmology 98.5 (1991): 766-785.

3. Photocoagulation treatment of proliferative diabetic retinopathy. "Clinical application of Diabetic Retinopathy Study (DRS) findings, DRS Report Number 8. The Diabetic Retinopathy Study Research Group". Ophthalmology 88.7 (1981): 583-600.

4. Photocoagulation treatment of proliferative diabetic retinopathy: relationship of adverse treatment effects to retinopathy severity. Diabetic retinopathy study report no. 5". Developments in Ophthalmology 2 (1981): 248-261.

5. Photocoagulation treatment of proliferative diabetic retinopathy: the second report of diabetic retinopathy study findings". Ophthalmology 85.1 (1978): 82-106.

6. Subash M., et al. "The Effect of Multispot Laser Panretinal Photocoagulation on Retinal Sensitivity and Driving Eligibility in Patients With Diabetic Retinopathy". JAMA Ophthalmology 134.6 (2016): 666-672.

7. Fong DS., et al. "Comparison of the modified Early Treatment Diabetic Retinopathy Study and mild macular grid laser photocoagulation strategies for diabetic macular edema". Archives of Ophthalmology 125.4 (2007): 469-480.

8. Scott IU., et al. "Effect of focal/grid photocoagulation on visual acuity and retinal thickening in eyes with non-center-involved diabetic macular edema". Retina 29.5 (2009): 613-617.

9. Vujosevic S., et al. "Microperimetry and fundus autofluorescence in diabetic macular edema: subthreshold micropulse diode laser versus modified early treatment diabetic retinopathy study laser photocoagulation". Retina 6 (2010): 908-916. 
10. Kim JT., et al. "Changes in choroidal thickness in relation to the severity of retinopathy and macular edema in type 2 diabetic patients". Investigative Ophthalmology and Visual Science 54.5 (2013): 3378-3384.

11. Zhu Y., et al. "Changes in choroidal thickness after panretinal photocoagulation in patients with type 2 diabetes". Retina 35.4 (2015): 695-703.

12. Entezari M., et al. "Choroidal Thickness in Healthy Subjects". Journal of Ophthalmic and Vision Research 13.1 (2018): 39-43.

13. Roohipoor R., et al. "Subfoveal Choroidal Thickness after Panretinal Photocoagulation with Red and Green Laser in Bilateral Proliferative Diabetic Retinopathy Patients: Short Term Results". Journal of Ophthalmology (2016): 9364861.

14. Nieves-Moreno M., et al. "New Normative Database of Inner Macular Layer Thickness Measured by Spectralis OCT Used as Reference Standard for Glaucoma Detection". Translational Vision Science and Technology 7.1 (2018): 20.

15. Muqit MMK., et al. "Effects of conventional argon panretinal laser photocoagulation on retinal nerve fibre layer and driving visual fields in diabetic retinopathy". Eye 24.7 (2010): 1136-1142.

16. Lee HJ., et al. "Long-term Effect of Panretinal Photocoagulation on Spectral Domain Optical Coherence Tomography Measurements in Diabetic Retinopathy". Current Eye Research 42.8 (2017): 1169-1173.

17. Demirok G., et al. "Changes in the Macular Ganglion Cell Complex Thickness and Central Macular Thickness after Argon Laser Panretinal Photocoagulation". Seminars in Ophthalmology 32.6 (2017): 759-763.

18. Kim JJ., et al. "One-year follow-up of macular ganglion cell layer and peripapillary retinal nerve fibre layer thickness changes after panretinal photocoagulation". The British Journal of Ophthalmology 98.2 (2014): 213-217.

19. Yang HS., et al. "Quantitative analysis of neural tissues around the optic disc after panretinal photocoagulation in patients with diabetic retinopathy". PloS one 12.10 (2017): e0186229.

20. Wadhwani M., et al. "Long term effect of panretinal photocoagulation on retinal nerve fiber layer parameters in patients with proliferative diabetic retinopathy". Oman Journal of Ophthalmology 12.3 (2019): 181-185.

21. Wadhwani M., et al. "A meta-analysis to study the effect of pan retinal photocoagulation on retinal nerve fiber layer thickness in diabetic retinopathy patients". Romanian Journal of Ophthalmology 64.1 (2020): 8-14.

22. Yun SH and Adelman RA. "Recent developments in laser treatment of diabetic retinopathy". Middle East African Journal of Ophthalmology 22.2 (2015): 157-163.

\section{Assets from publication with us}

- Prompt Acknowledgement after receiving the article

- Thorough Double blinded peer review

- Rapid Publication

- Issue of Publication Certificate

- High visibility of your Published work

Website: www.actascientific.com/

Submit Article: www.actascientific.com/submission.php

Email us: editor@actascientific.com

Contact us: +919182824667 